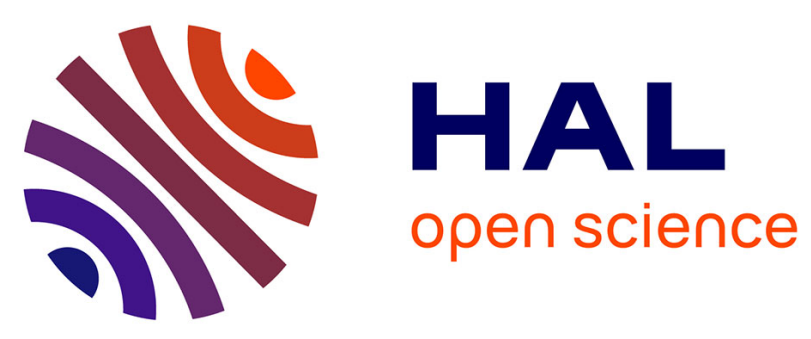

\title{
Spectral diffusion in glasses
}

\author{
R. Rammal, R. Maynard
}

\section{To cite this version:}

R. Rammal, R. Maynard. Spectral diffusion in glasses. Journal de Physique Lettres, 1978, 39 (12), pp.195-198. 10.1051/jphyslet:019780039012019500 . jpa-00231477

\section{HAL Id: jpa-00231477 https://hal.science/jpa-00231477}

Submitted on 1 Jan 1978

HAL is a multi-disciplinary open access archive for the deposit and dissemination of scientific research documents, whether they are published or not. The documents may come from teaching and research institutions in France or abroad, or from public or private research centers.
L'archive ouverte pluridisciplinaire HAL, est destinée au dépôt et à la diffusion de documents scientifiques de niveau recherche, publiés ou non, émanant des établissements d'enseignement et de recherche français ou étrangers, des laboratoires publics ou privés. 


\author{
Classification \\ Physics Abstracts \\ $61.40-63.20-62.80$
}

\title{
SPECTRAL DIFFUSION IN GLASSES
}

\author{
R. RAMMAL and R. MAYNARD \\ Centre de Recherches sur les Très Basses Températures, \\ C.N.R.S., B.P. $166 \mathrm{X}, 38042$ Grenoble Cedex, France
}

(Reçu le 28 février 1978, révisé le 24 avril 1978, accepté le 26 avril 1978)

\begin{abstract}
Rézumé. - Il est proposé un nouveau mécanisme de diffusion spectrale dans les verres, basé sur le processus de flip-flop entre 2 systèmes à 2 niveaux, dont les énergies de séparation sont différentes. Deux phonons sont nécessaires pour ce couplage : l'un réel pour conserver l'énergie et l'autre virtuel pour assurer le couplage élastique entre les deux défauts. Les constantes de diffusion spectrale et spatiale apparaissent comme paramètres d'une équation de Fokker-Planck. Une comparaison avec les processus plus conventionnels de bruit est esquissée.
\end{abstract}

\begin{abstract}
A new mechanism of spectral diffusion in glasses is proposed based on the flip-flop process between unlike defects. Two phonons participate in the process : one real phonon for conserving the energy and one virtual phonon for mediating the elastic interaction between the defects. The spectral-spatial diffusion is described in terms of a Fokker-Planck equation and a comparison with the classical noise process is discussed.
\end{abstract}

1. Introduction. - The existence of two level defects in glasses has been established by acoustic experiments at low temperature [1]. The energy splitting of these defects is broadly distributed over about $1 \mathrm{~K}$, as shown by the specific heat [2]. The transitions between the levels involve absorption or emission of phonons and the coupling energy with strain is usually very strong typically of the order of $1 \mathrm{eV}$ [1]. The problem of the elastic interaction between the two level defects has been discussed in detail by Joffrin and Levelut [3] and recent experiments of burning hole [4], phonon echoes [5] and dielectric echoes [6], confirm the presence of such interactions. From the one phonon direct coupling, two different effective interactions must be considered :

- the inhomogeneous interaction of the type $S_{1}^{z} S_{2}^{z}$ between the unlike defects 1 and 2 (i.e. defects whose splitting energies are different);

- the homogeneous or flip-flop interaction of the type $S_{1}^{x} S_{2}^{x}$ which couples only like defects 1 and 2 (the two level defects at site $R_{i} \overline{\text { can }}$ be represented by an effective spin $S_{i}$ of $\frac{1}{2}$ ).

The first type of interaction has been recognized as being dominant since all the defects can contribute to this static coupling, while only a very small fraction ( $\simeq 10^{-6}$ ?) of all the defects (the like spins) can participate in the homogeneous interaction. A calculation of the spectral diffusion has been done by Black and
Halperin [7] using a $S_{i}^{z} S_{j}^{z}$-interaction and the suddenjump model of Klauder and Anderson [8].

The purpose of this letter is to derive a new mechanism of spectral diffusion based on a phonon assisted flip-flop process.

In the first part, we will derive the transfer rate in terms of the mismatch energy and the relative distance between two defects. In the second part, we establish the kinetic equation of the energy transfer among the defects for which we derive the spectral and spatial diffusion constant.

2. Phonon assisted transfer rate. - Due to the mismatch energy $E_{2}-E_{1} \neq 0$, one real phonon is necessary in order to supply the mismatch energy $E_{2}-E_{1}$ (Fig. 1).

A similar situation occurs in the radiative transfer problem for inhomogeneously broadened optical lines [9]. However in this case, the interaction between the active ions is induced by the photon field.

We consider two different Hamiltonians at the two sites 1 and 2 :

- at site 1 , the two phonon (or Raman) coupling Hamiltonian [10] $\mathscr{H}_{1}=B_{1} S_{1}^{x} \varphi_{1} \varphi_{1}$;

- at site 2 , the standard one phonon (or direct) coupling Hamiltonian $\mathscr{H}_{2}=B_{2} S_{2}^{x} \varphi_{2}$.

Here, $\varphi_{1}$ (or $\varphi_{2}$ ) is the phonon field operator at site 1 (or 2), and $B_{1}$ and $B_{2}$ the coupling energy. 

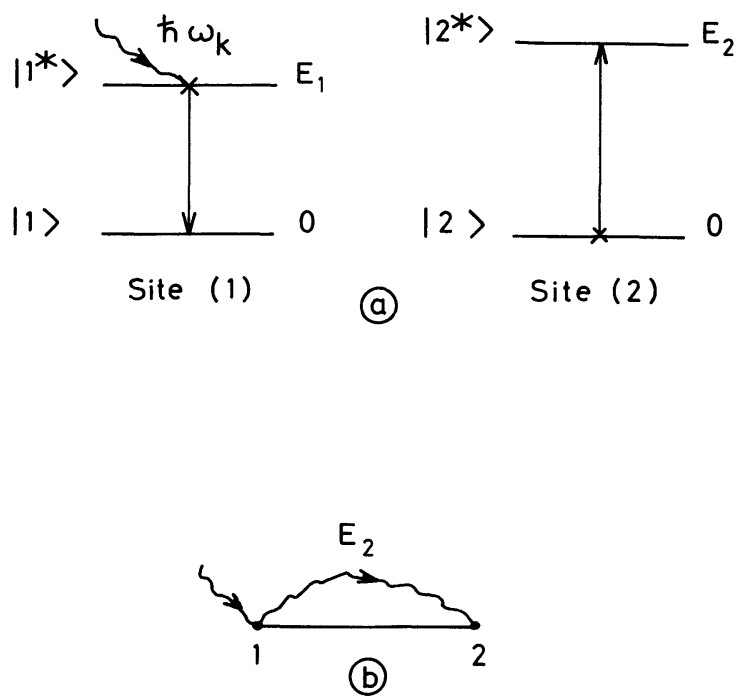

FIG. 1. - Mechanism of energy transfer between two unlike defects $\left(E_{2}>E_{1}\right)$ assisted by one phonon. $\left.a\right)$ In the initial state, the defect 1 is in the excited level of energy $E_{1}$ and the defect 2 is in its ground state. In the final state, the defect 1 is disexcited while the defect 2 is excited at $E_{2}$. In order to achieve the total energy conservation during this transition, a real phonon is absorbed by the defect 1 with $\hbar \omega_{\mathrm{k}}=E_{2}-E_{1}$ (the mismatch energy). b) The diagram (b) exhibits the phonons which participate to the mechanism : the real phonon $\hbar \omega_{\mathrm{k}}$, the exchanged virtual phonon of frequency $E_{2}$, mediating the elastic coupling between defects. This diagram shows the necessity of considering two-phonon (or Raman) coupling for the defect 1 and one phonon (or direct) coupling for the defect 2 .

The lowest-finite perturbation term is in fourth order in the coupling constant $B_{1}^{2} B_{2}^{2}$. The details of the calculation of the transition rate are too long to be reported in this letter, but they are straightforward : the expression for the transfer rate of excitation from 1 to 2 has been obtained :

$$
\begin{array}{r}
W\left(E_{2} R_{2} / E_{1} R_{1}\right)=\frac{32 \pi}{\hbar} B^{4} \frac{\hbar \omega_{\mathrm{k}}}{2 M v^{2}}\left|N\left(\frac{\hbar \omega_{\mathrm{k}}}{k_{\mathrm{B}} T}\right)\right| \times \\
\times\left|D\left(E_{2} \mathbf{R}_{12}\right)\right|^{2} \delta\left(\hbar \omega_{\mathrm{k}}-\left(E_{2}-E_{1}\right)\right)
\end{array}
$$

$W\left(E_{2} R_{2} / E_{1} R_{1}\right)$ is the probability (per unit time) of the flip-flop process of figure $1 ; B$ comes in from the simplification $B_{1}=B_{2}=B$ and $B^{4}$ indicates that this expression has been obtained from 4 th order perturbation theory in the coupling constant; $\omega_{\mathbf{k}}$ is the frequency of the phonon $\mathrm{k}, M$ the average mass of the atoms and $v$ the sound velocity in the approximation of a unique phonon branch; the factor $\hbar \omega_{\mathrm{k}} / 2 M v^{2}$ shows that the coupling between the spin and the phonons occurs through the local deformation; $N\left(\hbar \omega_{\mathbf{k}} / k_{\mathrm{B}} T\right)$ is the Bose-Einstein factor; $D\left(E_{2}, \mathbf{R}_{12}\right)$ is the phonon propagator over the distance $\mathbf{R}_{12}$ at the frequency $E_{2}$, describing the exchange of a virtual phonon between the spin 1 and 2 giving rise to the elastic interaction.

This formula can describe the general case where the mismatch energy $E_{2}-E_{1}=\Delta E$ can be positive or negative, corresponding to absorption (Fig. 1) or emission of the real phonon by defect 1 . In the case where $\Delta E=\hbar \omega_{\mathbf{k}}$ is negative the absolute value of the Bose-Einstein factor reestablishes the correct BoseEinstein factor $1+N\left(\frac{\hbar \omega_{\mathrm{k}}}{k_{\mathrm{B}} T}\right)$.

In this mechanism, there is no decoupling between the direct process responsible for the emission or absorption of a real phonon at one site - from which originates the spin-lattice relaxation $T_{1}-$ and the elastic interaction via the virtual phonon field between the two defects. It is important to notice this difference between our mechanism and the decoupling assumption used by Black and Halperin [7] in their calculation.

The general expression of the phonon propagator is rather complicated, except in the asymptotic regime of $q_{2} R_{12} \ll 1$ or $q_{2} R_{12} \gg 1$ where $q_{2}=\frac{E_{2}}{\hbar v}$. In glasses, the absolute concentration of the two level defects is not known since only a fraction of them are observed in the specific heat (typically $\gtrsim 10^{-6}$ ). As typically $q_{2} \lesssim \frac{k_{\mathrm{B}} T}{\hbar v} \simeq 10^{5} \mathrm{~cm}^{-1}$ at $1 \mathrm{~K}$ and $R_{12} \lesssim 10^{-6} \mathrm{~cm}$ for a relative concentration larger than $10^{-6}$, one gets $q_{2} R_{12} \lesssim 10^{-1}$, which justifies the use of the low frequency regime. In this case a very simple expression for the phonon propagator can be written in the acoustical phonon approximation :

$$
D\left(E_{2}, R_{12}\right) \simeq \frac{1}{4 \pi \rho v^{2}} \frac{1}{R_{12}^{3}} \text { for } q_{2} R_{12} \ll 1 .
$$

It is interesting to note here that this spatial variation comes directly from the scaling relation of the propagator :

$$
D\left(\mu^{-1} E_{2} ; \mu R_{12}\right)=\mu^{-3} D\left(E_{2}, R_{12}\right)
$$

where $\mu$ is a real scalar.

This behaviour in $R_{12}^{-3}$ seems to be very general in this limit, although in some cases $D=0$ can occur in perfect crystal due to high local symmetry [11]. A detailed discussion of this point is given in references [3], [11] and [12]. This spatial variation reflects the strong analogy between the elastic interaction and the electrical dipole-dipole interaction.

Within this regime, it has been verified that no cancellation occurs when the symmetrical process a two phonon coupling at site 2 instead of $1-$ is taken into account. It is in the next order of perturbation expansion that a destructive interference between these two processes can cancel this contribution [9].

By summing over all the phonons of wave vector $\mathbf{k}$, the transition rate [1] becomes proportional to the spectral density of phonons. In the acoustic approximation, this density is proportional to $\Delta E^{2 \cdot}$ and by 
using the simplified expression (2) of the propagator, (1) becomes :

$$
W\left(\Delta E, R_{12}\right) \approx \frac{|\Delta E|^{3}}{\left(R_{12}\right)^{6}}|N(\Delta E)|
$$

where $W\left(\Delta E, R_{12}\right)$ is the probability of transferring an energy $\Delta E$ between two defects. This expression is indeed valid both for $\Delta E \gtrless 0$. It is may be worth noting that $W(\Delta E)$ is an asymmetrical function of $\Delta E$, since the mechanism of energy transfer is assisted by one phonon. Moreover the maximum of $W(\Delta E)$ occurs at $\Delta E \simeq k_{\mathrm{B}} T$, the energy of the dominant phonons at temperature $T$.

3. Spectral-spatial diffusion. - Energy transfer between defects causes the rearrangement of the population distribution of the defects when they were initially disturbed. The present approach is based on the configuration averaging of the transport equations, following the procedure described by Vugmeister [13]. Let us consider a set of two level defects at point $\mathbf{R}_{i}$ and with a splitting energy $E_{i}$ $\left(x_{i}\right.$ is a four-coordinate vector $\left.\bar{x}_{i}=\left(\mathbf{R}_{i}, E_{i}\right)\right)$. The number of defects in their excited state at equilibrium is $N_{i}$ while the deviation from $N_{i}$ at time $t$ is $\delta N_{i}(t)$. The kinetics equation starts from the master equation

$$
\frac{\partial \delta N_{i}}{\partial t}=-\sum_{j} W_{i j} \delta N_{i}+\sum_{j} W_{j i} \delta N_{j}
$$

where the defect-lattice relaxation process of type $T_{1}$ has been neglected for simplicity.

The time development of a non-equilibrium population is completely described by the mean density of excitations $\rho(\bar{x})$ averaged over the spectral and spatial distribution of defects in the $(\mathbf{R}, E)$ space :

$$
\begin{gathered}
\rho(\bar{x}, t)=\left\langle\sum_{i} \delta\left(\bar{x}-\bar{x}_{i}\right) \delta N_{i}\right\rangle \\
\langle[\ldots]\rangle= \\
=\frac{1}{V^{N}} \int \mathrm{d} x_{1} \mathrm{~d} x_{2} \ldots \mathrm{d} x_{N} n\left(E_{1}\right) n\left(E_{2}\right) \ldots n\left(E_{N}\right)[\ldots]
\end{gathered}
$$

where $V$ is the volume of the sample and $n(E)$ the energy density of two level defects. A uniform and random distribution of the defects has therefore been assumed as has the absence of correlations between the spatial and energy distributions. Moreover the following calculation is only valid when the width of $n(E)$, i.e. $E_{\mathrm{MAX}}$, is larger than the width of $W(\Delta E)$ of (3), which is of the order of $k_{\mathrm{B}} T$; which implies $k_{\mathrm{B}} T \ll E_{\mathrm{MAx}}$.

To exhibit the characteristic time $\tau$ in the master equation (4), which plays the role of a cross relaxation time, let us first neglect the back transfer $\sum_{j} W_{j i} \delta N_{j}$ in (4). Then the solution of (4) can be written in term of $Q_{i}(t)=\exp \left\{-\sum_{j} W_{i j} t\right\}$ where $Q_{i}(t)$ is the pro- bability that the defect $i$ remains in its excited state during the time $t$. The average of $Q_{i}$ over configurations defined by (6) gives :

$$
Q(t)=\exp \left\{-\int \mathrm{d} x n(E)\left(1-\mathrm{e}^{-W(x) t}\right)\right\} .
$$

The time dependence of $Q(t)$ is controlled by the spatial variation of $W$ in $R^{-6}$. For a constant energy density, $n(E)=n / E_{\mathrm{MAX}}$ up to $E_{\mathrm{MAX}},(n=$ density of defects per volume unit) and we get directly

$$
Q(t)=\exp \{-\sqrt{t / \tau}\} .
$$

A simple expression for $\tau$ can be written if one puts (3) in the form of $W=\xi f(R) g(\Delta E)$ where $f(R)=R^{-6}$

$$
\begin{aligned}
& \text { and } g(\Delta E)=|\Delta E|^{3}\left|N\left(\frac{\Delta E}{k_{\mathrm{B}} T}\right)\right| ; \text { then : } \\
& \tau^{-1 / 2}=\frac{n \pi^{3 / 2}}{12 E_{\mathrm{MAX}}} \xi^{1 / 2} \int \sqrt{g(\Delta E)} \mathrm{d} E^{\prime} .
\end{aligned}
$$

In the general case, the back transfer must be taken into account. In the limit of $t \gg \tau$, we have derived the differential version of (4) which is identified as a Fokker-Planck equation for the density of excitation $\rho(\bar{x}, t)$ :

$$
\frac{\partial \rho(\bar{x} t)}{\partial t}=-K^{\prime} \frac{\partial \rho(\bar{x} t)}{\partial E}+K \frac{\partial^{2} \rho(\bar{x} t)}{\partial E^{2}}+D \bar{\nabla} \frac{2}{R} \rho(\bar{x}, t)
$$

where :

$$
\begin{aligned}
D & =\frac{1}{2 \tau}(\bar{n})^{-2 / 3}\left[\int \mathrm{d} E^{\prime}(g(\Delta E))^{5 / 6}\right] \times \\
\times & {\left[\int \mathrm{d} E^{\prime} \sqrt{g(E)}\right]^{-5 / 3} } \\
K=\frac{1}{4 \tau} & {\left[\int \mathrm{d} E^{\prime}(\Delta E)^{2} \sqrt{g(\Delta E)}\right]\left[\int \mathrm{d} E^{\prime} \sqrt{g(\Delta E)}\right]^{-1} } \\
K^{\prime}=\frac{1}{2 \tau} & {\left[\int \mathrm{d} E^{\prime}(\Delta E) \sqrt{g(\Delta E)}\right]\left[\mathrm{d} E^{\prime} \sqrt{g(\Delta E)}\right]^{-1} }
\end{aligned}
$$

and

$$
\Delta E=E-E^{\prime} .
$$

In this calculation we have neglected the defectlattice relaxation rate $T_{1}^{-1}$. Equation (10) is therefore only valid for the condition that $\tau$ is shorter than the shortest $T_{1}$ of the defect i.e. $\tau \ll t \ll T_{1}$. In (10), $D$ is the spatial diffusion while $K$ is the spectral diffusion. The occurrence of a drift term $K^{\prime}$ in (10) is due to the asymmetry of $W$ as a function of $\Delta E$, which is absent in reference [13].

As we are mainly interested in the spectral diffusion instead of spatial diffusion, we can derive directly the Green's function solution of (10) as a response of an infinitely narrow pulse of frequency $E_{0} / h$ :

$$
\begin{array}{r}
\rho(E, t)=\frac{1}{\sqrt{4 \pi K t}} \exp \left[-\frac{\left(E-E_{0}-K^{\prime} t\right)^{2}}{4 K t}\right] \\
(t>0) .
\end{array}
$$


The line shape is a Gaussian of width $\sqrt{2 K t}$; its height decreases in time as $1 / \sqrt{4 \pi K t}$ and its centre moves at the velocity $K^{\prime}$ (energy/time).

The characteristic time of this sort of cross over relaxation process is now calculated from (9) by reestablishing the parameters of the transfer energy rate (1) and (2) in the approximation of a single acoustic branch of Debye temperature $\theta_{\mathbf{D}}$ :

$$
\tau^{-1}=\frac{10^{3} B^{4} T^{5} C^{2}}{\hbar\left(m v^{2}\right)^{3} \theta_{\mathrm{D}}^{3} E_{\mathrm{MAX}}^{2}},
$$

where $C$ is the relative concentration of defects.

From the expressions (10) of $D, K$ and $K^{\prime}$, we get directly :

$$
\begin{array}{rlrl}
K & \simeq 8.5 \frac{T^{2}}{\tau} & \text { in }(\mathrm{K})^{2} \mathrm{~s}^{-1} \\
K^{\prime} \simeq 2.5 \frac{T}{\tau} & \text { in }(\mathrm{K}) \mathrm{s}^{-1} \\
D & =\frac{0.5}{\tau}\left(\frac{E_{\mathrm{MAX}}}{n T}\right)^{2 / 3} & \text { in }\left(\mathrm{cm}^{2} . \mathrm{s}^{-1}\right)
\end{array}
$$

These temperature power laws originate in the BoseEinstein factor in $g$ which indicates that $\Delta E$ is scaled by $k_{\mathrm{B}} T$ in the integrals. The power in $T$ are therefore $T^{7}$ for $K, T^{6}$ for $K^{\prime}$ and $T^{4.33}$ in $D$. They reflect rather strong variation in temperature of the spectral-spatial diffusion constant making the effect unobservable at too low a temperature.

The interval of time in which this spectral diffusion can be observed is defined by $\tau \ll T_{1} . T_{1}$, the defectlattice relaxation time is well known : for defects with energy $E \ll k_{\mathrm{B}} T$, the expression of $T_{1}^{-1}$ is $(\pi / \hbar) B^{2} E^{2} T / \theta_{\mathrm{D}}^{3}\left(m v^{2}\right)$ and its value is typically $1 \mu \mathrm{s}$ at $1 \mathrm{~K}$. It is then possible to form the ratio $T_{1} / \tau$ and compare to 1 :

$$
\frac{T_{1}}{\tau}=50 \frac{T^{4} B^{2} C^{2}}{E^{2}\left(m v^{2}\right)^{2} E_{\mathrm{MAX}}^{2}} .
$$

This ratio shows that the window in time for this effect can be opened at low energy $E$ or moderate temperature. With the typical value for $\mathrm{SiO}_{2}$ glass : $C=10^{-3}$; $E_{\mathrm{MAX}}=100 \mathrm{~K} ; \quad n=10^{20} \mathrm{~cm}^{-3} ; \quad m v^{2}=10^{4} \mathrm{~K}$, $B=10^{4} \mathrm{~K}$ and $E=10^{-4} \mathrm{~K}$, one gets $T_{1} / \tau \simeq T^{4}$ ( $T$ in $\mathrm{K}$ ) which indicates that this process can operate around $1 \mathrm{~K}$. With these numbers, the typical values are : $\tau=10^{-6} \mathrm{~s} \quad($ at $1 \mathrm{~K}) ; \quad K=10^{7} \mathrm{~K}^{2} \mathrm{~s}^{-1}$; $K^{\prime}=10^{6} \mathrm{~K} \mathrm{~s}^{-1} ; D=10^{-6} \mathrm{~cm}^{2} \cdot \mathrm{s}^{-1}$ (at $1 \mathrm{~K}$ ).

Finally a Gaussian line is predicted by this mechanism in the burning hole experiment instead of a Lorentzian line as in the Black and Halperin [7] model. For the Gaussian profile of the line the height evolves in time and temperature as

$$
(2 \pi K t)^{-1 / 2} \approx T^{-7 / 2} t^{-1 / 2}
$$

instead of $T^{-4} t^{-1}$ for the Lorentzian line. Therefore one can expect this mechanism to become dominant at longer times than the noise process of reference [7].

Acknowledgment. - The authors wish to thank Professor R. Orbach for drawing their attention to the strong similarity between this problem and the radiative transfer problem for inhomogeneously broadened optical lines.

\section{References}

[1] Hunklinger, S. and Arnold, W., Physical Acoustics, Ed. W. P. Mason and R. N. Thurston (Academic Press) 1976, Vol. XII, p. 155.

[2] Lasjaunias, J. C., Ravex, A., Thoulouze, D. and VaNDORPE, M., International Conference of Phonon Scattering in Solids (Plenum Press) 1975, p. 138.

[3] Joffrin, J., Levelut, A., J. Physique 36 (1975) 811.

[4] Arnold, W., Hunklinger, S., Solid State Commun. 17 (1975) 883.

[5] Golding, B., Graebner, J., Phys. Rev. Lett. 37 (1976) 852.

[6] Bernard, L., Piche, L., Schumacher, G., Joffrin, J., GraebNER, J., J. Physique Lett., à paraître.
[7] Black, J. L., Halperin, B. I., Phys. Rev. B 16 (1977) 2879.

[8] Klauder, J. R., Anderson, P. W., Phys. Rev. 125 (1962) 912.

[9] Holstein, T., Lyo, S. K. and OrbaCH, R., Phys. Rev. B 16 (1977) 934.

[10] In order to obtain a flip-flop transition by means of one phonon couplings alone it is necessary to introduce a static coupling in $B S^{z} \varphi$. This mechanism would led to a contribution in $B^{6}$ at least and it will be discarded here.

[11] Orbach, R., TACHIKI, M., Phys. Rev. 158 (1967) 524.

[12] MCMahon, D., Silsbee, R., Phys. Rev. A 135 (1964) 91.

[13] Vugmeister, B. E., Sov. Phys. Solid State 18 (1976) 469. 